\title{
Genomic and proteomic evidence supporting the division of the plant pathogen Ralstonia solanacearum into three species
}

Philippe Prior ${ }^{1,4^{*}+}$, Florent Ailloud ${ }^{1,2+}$, Beth L. Dalsing ${ }^{3}$, Benoit Remenant ${ }^{1}$, Borja Sanchez ${ }^{4,5}$ and Caitilyn Allen ${ }^{3}$

\begin{abstract}
Background: The increased availability of genome sequences has advanced the development of genomic distance methods to describe bacterial diversity. Results of these fast-evolving methods are highly correlated with those of the historically standard DNA-DNA hybridization technique. However, these genomic-based methods can be done more rapidly and less expensively and are less prone to technical and human error. They are thus a technically accessible replacement for species delineation. Here, we use several genomic comparison methods, supported by our own proteomic analyses and metabolic characterization as well as previously published DNA-DNA hybridization analyses, to differentiate members of the Ralstonia solanacearum species complex into three species. This pathogen group consists of diverse and widespread strains that cause bacterial wilt disease on many different plants.
\end{abstract}

Results: We used three different methods to compare the complete genomes of 29 strains from the $R$. solanacearum species complex. In parallel we profiled the proteomes of 73 strains using Matrix-Assisted Laser Desorption/lonization-Time of Flight Mass Spectrometry (MALDI-TOF-MS). Proteomic profiles together with genomic sequence comparisons consistently and comprehensively described the diversity of the $R$. solanacearum species complex. In addition, genome-driven functional phenotypic assays excitingly supported an old hypothesis (Hayward et al. (J Appl Bacteriol 69:269-80, 1990)), that closely related members of the $R$. solanacearum could be identified through a simple assay of anaerobic nitrate metabolism. This assay allowed us to clearly and easily differentiate phylotype II and IV strains from phylotype I and III strains. Further, genomic dissection of the pathway distinguished between proposed subspecies within the current phylotype IV. The assay revealed large scale differences in energy production within the $R$. solanacearum species complex, indicating coarse evolutionary distance and further supporting a repartitioning of this group into separate species.

Conclusions: Together, the results of these studies support the proposed division of the $R$. solanacearum species complex into three species, consistent with recent literature, and demonstrate the utility of proteomic and genomic approaches to delineate bacterial species.

Keywords: Ralstonia solanacearum, Bacterial wilt, Plant pathogen, Taxonomy, Genomics, Proteomics

\footnotetext{
*Correspondence: philippe.prior@cirad.fr

${ }^{\dagger}$ Equal contributors

${ }^{1}$ UMR PVBMT Peuplements Végétaux et Bioagresseurs en Milieu Tropical,

CIRAD, Saint Pierre, La Réunion, France

${ }^{4}$ Department of Plant Health and Environment (SPE), INRA, Paris, France

Full list of author information is available at the end of the article
} 


\section{Background}

Thousands of genetically distinct strains within the Ralstonia solanacearum species complex (RSSC) cause bacterial wilt diseases in plants. These bacteria colonize the xylem tissue of host plant vascular systems causing stunting, wilting, yield reduction, and death. This pathogen group has major economic and social impact worldwide [2, 3]. Members of the RSSC can collectively infect over 250 hosts in 54 botanical families and include: $R$. solanacearum strains, which collectively infect a broad host range and are typically soil-borne; R. syzygii, a spittlebug-transmitted pathogen that causes Sumatra disease in cloves; and the Blood Disease Bacterium (BDB), an unclassified organism responsible for the pollinator-transmitted Blood Disease of bananas and plantains in the Philippines.

Smith first described the morphological and chemotaxonomic characteristics of the bacterial wilt pathogen as Bacterium solanacearum, and this species has most recently been placed in the genus Ralstonia $[4,5]$. The BDB was described and named Pseudomonas celebensis in 1921 $[6,7]$. However this name lost its standing in nomenclature when the original strain was lost so there was no authentic type strain. The Sumatra disease pathogen, originally described as Pseudomonas syzygii, was placed in a separate species in the genus Ralstonia based on $16 \mathrm{~S}$ sequences and DNA-DNA hybridization (DDH) data showing substantial divergence from $R$. solanacearum [8]. However, the DDH study that concluded $R$. syzygii should be placed in a separate species was based on a comparison with $R$. solanacearum $K 60^{\mathrm{T}}$, a phylotype II strain that is quite phenotypically and genotypically divergent from $R$. syzygii, a member of phylotype IV.

DNA-DNA hybridization has been used to distinguish species since the 1960s, contributing importantly to the modern bacterial species concept [7, 9]. However, because complete sequenced genomes contain significantly more information than can be inferred from the results of DDH and computer-driven methods are less prone to human error, this technique can now be replaced with bioinformatics methods [10, 11]. Early analyses based on the single-gene phylogeny of the conserved egl, mutS, $h r p B$ or ITS sequences divided the RSSC into four distinct genospecies, known as phylotypes, corresponding to strain geographic origin: phylotype I (Asia), phylotype II (Americas), phylotype III (Africa), and phylotype IV (Indonesia and Japan) [12-15]. The phylogenetic structure of the RSSC was subsequently confirmed in an extensive series of genomic studies involving a large array of analytical methods from microsatellites and MLST to microarrays $[13,16,17]$.

While the analyses of the complete genome sequences of several strains in the RSSC provides strong evidence supporting the phylotype structure [9, 12, 18-20], they further reveal a larger degree of evolutionary divergence among the phylotypes that warrants the division of the RSSC into three species, as previously suggested [19]. Recently, Safni et al., [21] supported this taxonomic revision, calling for an amendment of the descriptions of the RSSC based on a polyphasic approach with emphasis on DNA-DNA hybridization analysis. Safni suggested that R. pseudosolanacearum sp. nov., corresponding to phylotypes I and III, and Ralstonia syzygii, corresponding to phylotype IV should be considered two species. Based on differences in pathological phenotype, they suggested that $R$. syzygii be further divided into three subspecies. The broad host-range soil-borne strains were proposed to be renamed $R$. syzygii subspecies indonesiensis subsp. nov. The unclassified banana Blood Disease Bacterium was proposed to be named $R$. syzygii subspecies celebesensis subsp. nov. and $R$. syzygii, which causes Sumatra disease in cloves, was proposed to be renamed $R$. syzygii subspecies syzygii subsp. nov. Finally, Safni called for phylotype II strains (from the Americas), which include the species type strain $\mathrm{K}_{60}^{\mathrm{T}}\left(=\mathrm{ATCC} 11696^{\mathrm{T}}=\mathrm{LMG}^{\mathrm{T}} 299^{\mathrm{T}}\right)$, to remain in $R$. solanacearum.

In the present study, we used a combination of genomic and proteomic methods and a large genome pool to unambiguously delineate species within the RSSC. We formally examined these methods for their correspondence to the recent reclassification of this taxonomically disputed organism into three distinct species based on DNA-DNA hybridization. This work validates the redistribution of $R$. solanacearum into three species based on modern methods. We further provide tools for the rapid identification and classification of new isolates into species and subspecies without DNA-DNA hybridization.

\section{Results and discussion}

Phenotypic diversity in the RSSC

Safni et al., [21] analyzed the RSSC using phenotype microarrays and identified major variation in the core metabolisms both between and within phylotypes, which generally supported the idea that $R$. solanacearum can be divided into multiple species but no assay was able to provide clear distinction between the three proposed species. In the present study, we carefully dissected functional as well as genotypic differences in the denitrification metabolic pathway. This pathway is associated with several quantifiable and biologically relevant phenotypic traits that play major roles in virulence of a phylotype I strain [22]. Additionally, these phenotypes were known to vary among strains prior to modern-day genome sequencing and phylotyping [1].

In 1990, Hayward recognized variability in anaerobic nitrogen metabolism between $R$. solanacearum strains [23]. However, the biovar sub-classification system, in use at the time, did not correspond to the phylogenetic 
relationships among RSSC strains. No clear patterns differentiated biovars by anaerobic nitrogen metabolism, thus denitrification was not considered a useful trait for strain typing. Recent phylogenetic analyses that reclassified strains into phylotypes, and here into species, motivated us to revisit the idea that this metabolic trait could be used to differentiate phylogenetically distinct groups.

Denitrification is an anaerobic respiration process that allows strains to use nitrate as a terminal electron acceptor to grow under anaerobic conditions. Nitrate $\left(\mathrm{NO}_{3}^{-}\right)$is converted successively to nitrite $\left(\mathrm{NO}_{2}^{-}\right)$, nitric oxide (NO), nitrous oxide $\left(\mathrm{N}_{2} \mathrm{O}\right)$, and finally nitrogen gas $\left(\mathrm{N}_{2}\right)$ in a series of four reactions catalyzed by the products of the narG, aniA, norB and nos $Z$ genes, respectively. To determine which strains were able to use denitrification for energy and growth, we incubated 68 strains (14 phylotype I; 35 phylotype II; 11 phylotype III; 8 phylotype IV) anaerobically both with and without nitrate. After 72 h, we measured O.D. 600 and calculated the ratio of endpoint optical density in cultures provided with nitrate compared with those lacking a traditional terminal electron acceptor. Strains with a ratio equal to or above two were considered able to respire on nitrate under these conditions. All tested strains in phylotypes I and III respired on nitrate but no strains within phylotypes II and IV exhibited this trait (Fig. 1a).
Our phenotypic data on nitrogen metabolism in the RSSC conflict with those presented by Safni et al. Their formal proposed new and emended species descriptions state that "some" strains of phylotype II and "most" strains of the proposed $R$. syzygii subsp. indonesiensis in phylotype IV can reduce nitrate to nitrogen gas, while "most" strains of phylotypes I and III (proposed $R$. pseudosolanacearum) can reduce nitrate to nitrogen gas. In contrast, our biochemical assays of 68 diverse RSSC strains consistently found that no strains in phylotypes II and IV could complete denitrification under anaerobic conditions, while all strains in phylotypes I and III can do so. These contrasting results may reflect differences between our denitrification assays [22] and the 1964 Hayward method used by Safni et al.

The denitrification phenotypes were consistent with genomic analysis showing that all sequenced phylotype I and III strains have a gene (narG) encoding the major catalytic subunit of a respiratory nitrate reductase (Fig. 1b). Sequenced phylotype II strains do harbor narG, but the functional analysis described above, indicates that these strains either do not make a functional enzyme or do not use this nitrate reductase under the anaerobic conditions tested here. Phylotype IV strains are genomically divided into three groups based on presence or absence of the nitrate (narG), nitrite (aniA), and nitric

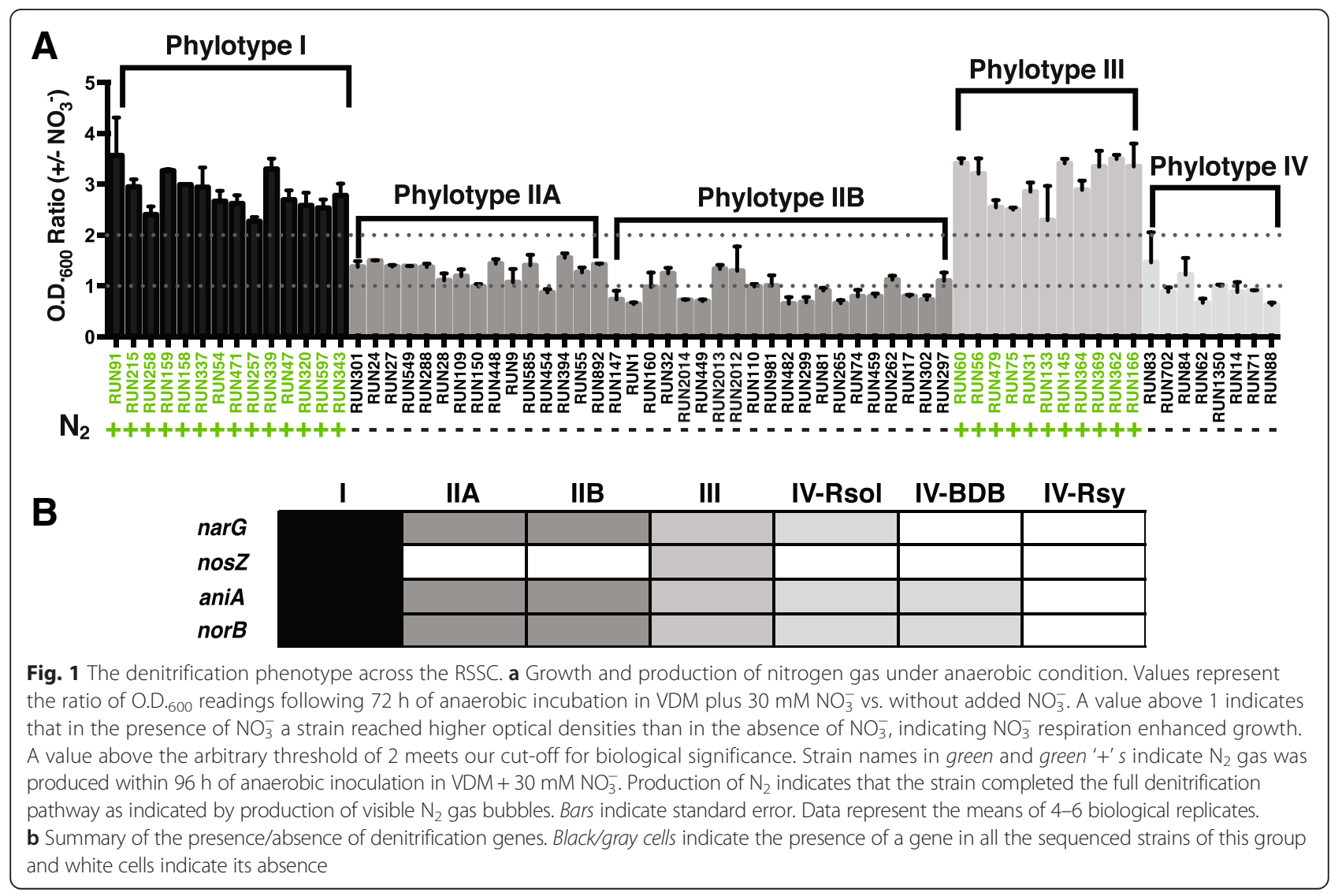


oxide (nor $B)$ reductase genes that encode the first three steps of the pathway. All sequenced strains of the proposed $R$. syzygii subspecies indonesiensis (currently phylotype IV $R$. solanacearum) contain narG, but strains of the proposed $R$. syzygii subspecies celebesensis (currently phylotype IV BDB) lack this gene. Strains of the proposed $R$. syzygii subspecies syzygii (currently phylotype IV $R$. syzygii) lack narG, aniA, and norB.

We used functional analyses to determine if each strain could denitrify completely. Complete denitrification, the full step-wise conversion of nitrate to dinitrogen, is indicated by the production of visible dinitrogen gas bubbles. Importantly, this gas was absent in all cultures that were not provided nitrate. Phylotypes I and III did complete denitrification, but no members of phylotypes II and IV did (Fig. 1a). This finding correlated perfectly with our genomic observation that all sequenced phylotype I and III strains contain nos $Z$, encoding a nitrous oxide reductase while no sequenced phylotype II or IV strains harbor nosZ (Fig. 1b).

From an evolutionary perspective, it appears that these three proposed species have adapted to use different energy production mechanisms. Phylotypes I and III use denitrification under anaerobic conditions for energy while phylotypes II and IV do not. Furthermore, phylotype II strains have maintained genes that suggest they regulate this metabolism differently. Phylotype IV strains appear to be in the process of losing this pathway altogether. This step-wise loss of denitrification may be associated with the ability to be vector transmitted, like $R$. syzygii subsp. syzygii. Alternatively, if the pathway is being gained stepby-step, denitrification may enhance soil survival and transmission via root entry. These hypotheses are being further explored. As Hayward proposed in 1990, groups in the RSSC can be distinguished all the way down to the subspecies level by a combination of functional and genomic evaluation of a single pathway, denitrification.

\section{Genomic diversity in the RSSC}

The 16S rRNA gene sequences of $R$. solanacearum strains are more than $97 \%$ identical, suggesting that this group forms a single species that is distinct from its close relative $R$. eutropha [5]. However, 16S rRNA sequences do not always accurately reflect similarities at the whole-genome level and they cannot distinguish between recently diverged species [24, 25]. Moreover, this identity threshold has not been universally accepted, and distinct species with $98 \%$ identical $16 \mathrm{~S}$ rRNA sequences have been described [23]. DDH was historically used for species delineation, and a $70 \%$ DNA-DNA similarity was traditionally used to define species. Recently, Safni et al. argued that $R$. solanacearum can be divided into 3 species based on DDH values. In a complementary approach, we evaluated the taxonomy of $R$. solanacearum using genomic and proteomic data. As a method, DDH has significant drawbacks: it is technically difficult, is performed only in a few specialized laboratories, and is prone to experimental errors [26]. DDH assays can only measure the potential for hybridization between purified DNA from two organisms, without regard to biological function. Thus, the $70 \%$ $\mathrm{DDH}$ criterion does not correspond to $70 \%$ shared orthologous genes or even $70 \%$ sequence identity [27]. Strains showing more than $70 \% \mathrm{DDH}$ can possess up to $21 \% \mathrm{di}-$ vergent gene content, which is equivalent to around 1000 genes in a typical 5.3 Mb $R$. solanacearum genome [28]. With the recent development of in silico comparative methods using complete genome sequences, $\mathrm{DDH}$ is no longer the most reliable method for determining relatedness between bacterial strains.

In a previous study [19], we proposed division of the RSSC into 3 genomospecies based on a genome-to-genome comparison using Average Nucleotide Identity (ANI) analyses of 8 strains. In the present study, we included 15 additional genomes in the ANI analysis and compared the ANI data to two other genomic analysis methods showing a better correlation with DDH: the Maximum Unique Matches index (MUMi) and the Genome-to-Genome Distance Calculator (GGDC) [11, 29-31] (Additional file 1).

Briefly, ANI detects the level of conservation or similarity of the total genomic sequences shared between two strains based on the identification of homologous fragments of fixed length using the BLAST algorithm. Strains with ANI $>95 \%$ are considered as belonging to the same species, consistent with the $70 \% \mathrm{DDH}$ criterion [11, 28, 30, 32-34]. Like DDH, ANI accounts for the variability in conserved gene content but does not always reflect differences between closely related strains and strains with similar ANI values can have similar or dissimilar gene content [35]. The MUMi algorithm overcomes this problem by accounting for both the variability of homologous gene content and the gain and loss of DNA. MUMi distances are derived from a list of maximum unique matches (MUMs) of a given minimal length shared between two genomes and the average length between genomes. Because this technique uses a fast algorithm to detect MUMs, MUMi is significantly faster than ANI. A MUMi value of $0.33 \pm 0.03$ corresponds to an ANI value of $95 \%$. Finally, the recently revised GGDC method shows the highest correlation with wet-lab DDH $[31,36]$. The GGDC also infers in silico $\mathrm{DDH}$ values from genomic distances; therefore, a similar $70 \%$ threshold can be used. Although this method is based on principles similar to ANI and MUMi, GGDC uses a different set of formulas to estimate genomic distances.

The ANI values obtained from pairwise comparisons between all genomes are presented in Additional file 1. The 29 strains analyzed in the present study fall into 
three distinct groups. The first group includes strains from phylotypes I and III. The second group comprises phylotype II strains, divided into subgroups IIA (containing the current $R$. solanacearum type strain, $\mathrm{K} 60^{\mathrm{T}}$ ) and IIB. The last group includes phylotype IV strains (PSI07, BDB R229 and $R$. syzygii R24), originally described as a separate species before the establishment of the species complex. These ANI results are wholly consistent with a previous analysis of a smaller group of genomes [19].

The genomic distances calculated using the MUMi algorithm are presented in Additional file 1. This method separates the RSSC into 3 or more species depending on how strictly the $0.33 \pm 0.03$ criterion is applied. Consistent with the ANI analysis, this method identifies two distinct species: one containing phylotype IV, and one containing phylotypes I and III. However, the delineation of phylotype II as a single species was not definitive. The genomic distances were $<0.33 \pm 0.03$ in $100 \%$ of the strains within subgroups IIA and IIB but some genomic distances were $>0.33 \pm 0.03$ between few strains of the two subgroups. Notably, the distances between IIB strain UW551 and 5 of the 6 IIA strains were above the threshold $(0.37-0.40)$. However, we believe this reflects the relatively low quality of the UW551 genome, and not true biological or genomic diversity.
In silico DDH values inferred using the GGDC algorithm are presented in Additional file 1. According to the traditional $70 \%$ DDH criterion, GGDC distinguishes 5 species within RSSC with phylotypes I, III and IV assigned to single species and phylotype II divided into 2 species corresponding to the IIA and IIB subgroups. Thus, GGDC more clearly differentiates between closely related strains. Although GGDC divides the RSSC into more species than ANI and MUMi, the raw result patterns are consistent between all 3 methods. As previously observed with the MUMi distances, IIA and IIB strains are definitely divergent at the whole-genome level. The ANI values between phylotypes I and III, or subtypes IIA and IIB, were approximately $96 \%$, while the ANI values within the species predicted using GGDC ranged from 97 to $99 \%$. To resolve these slightly varying analyses, we used SplitsTree software to build a phylogenetic network derived from the ANI, MUMi and GGDC distance matrices. The results showed no obvious ambiguities, confirming that all three genome sequence-based methods give broadly consistent results (Fig. 2).

The ANI, MUMi and GGDC methods are all based on whole-genome comparisons and have been shown to correlate well with the traditional standard method, DDH. Nonetheless, the RSSC can be divided into three to five species depending on how the genomic distances

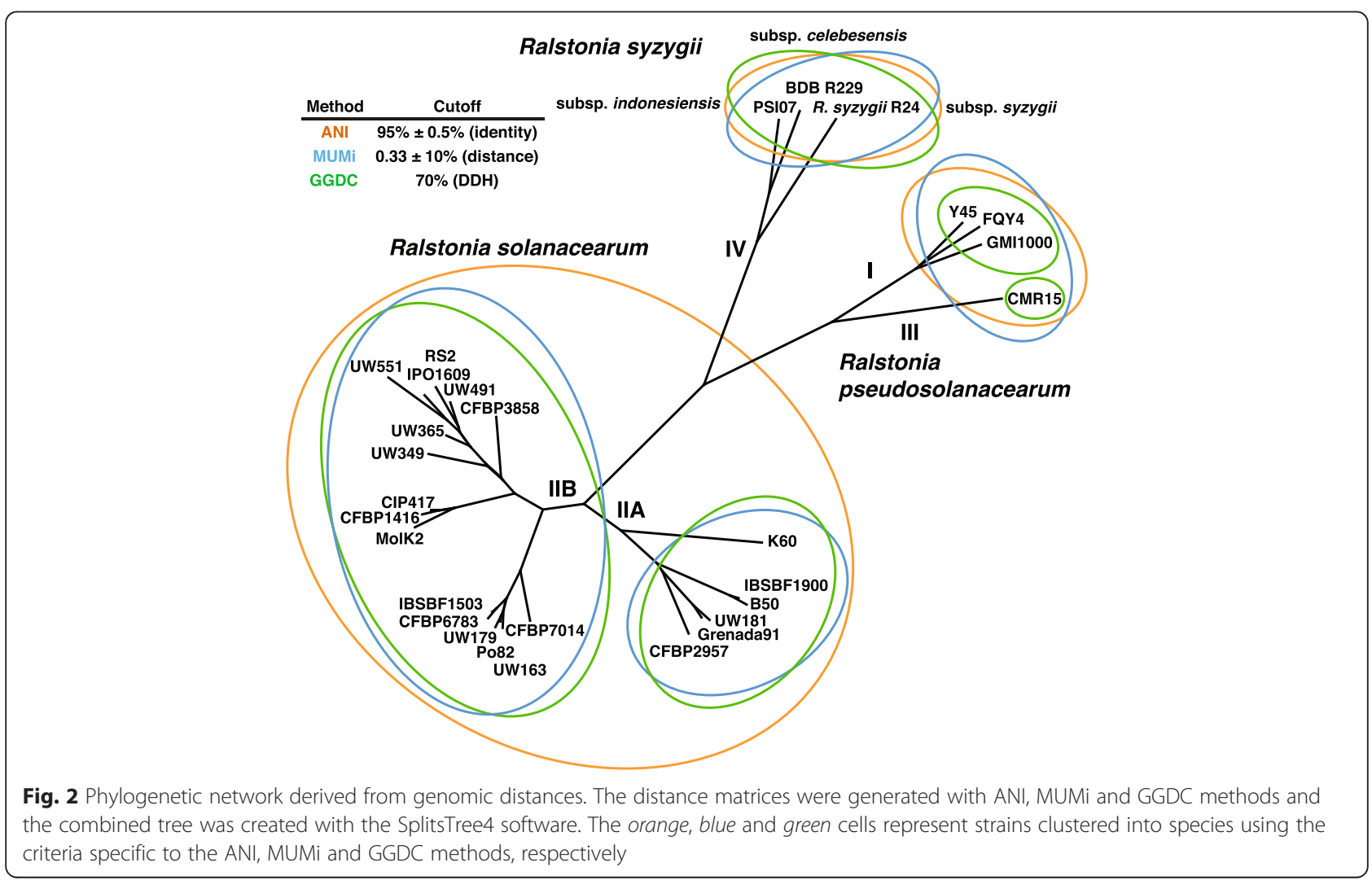


are calculated and the criteria used. Taken together, the outputs of these techniques illustrate the difficulty of consistently delineating species among closely related strains. Based on phenotypic data, we conclude that ANI and MUMi distances adequately reflect the level of biological variability within the RSSC, with a three-species division in which phylotypes I and III cluster together, and phylotypes II and IV are further apart.

\section{Proteomic diversity in the RSSC}

A total of 73 bacterial strains representing the four phylotypes were subjected to comparative proteomic analysis as a complementary method to the genome-based analyses presented above (Additional file 2). Protein mass spectra corresponding to each strain were obtained using MALDI-TOF and clustered using SPECLUST software [37]. This generated a list of common peaks represented as inter-sample consensus $\mathrm{m} / \mathrm{z}$ values. The best results were achieved using a "within peak match score $(\sigma)$ " of $3 \mathrm{Da}$, as defined in the SPECLUST documentation. The consensus spectra matrix was translated to a binary matrix in which the absence/presence of a consensus peak in all strain profiles was represented as 0 or 1 , respectively. This binary matrix was used to infer the phylogenetic relationships among the strains with the MALDI-TOF data and Bayesian analysis using MrBayes v3.2.2 software [38].
The MALDI-TOF approach was previously used for bacterial identification [39]. Mass fingerprinting is a simple, quick and reproducible method for bacterial identification through the generation of large spectral databases [40].

Taxonomically, molecular typing using protein profiles has been useful for bacterial classification at the species and subspecies levels $[41,42]$ and at the strain level, depending on the type and class of bacterial group considered [43]. In the present study, a combination of MALDI-TOF profiling, consensus mass peak lists, and Bayesian inference was used to cluster the 73 Ralstonia strains into three groups with strong branch support. The first cluster contained phylotypes I and III, whereas the second cluster contained phylotypes IIA and IIB, and the third cluster contained phylotype IV (Fig. 3). These results were consistent with the findings of the genomic analysis, supporting the division of $R$. solanacearum into three species.

\section{Phenotypic, genomic and proteomic data converge on a three-species model}

The taxonomic classification of $R$. solanacearum has changed repeatedly over the last 50 years, grouping strains with divergent ecological, geographical, genetic and phenotypic profiles, including many pathological

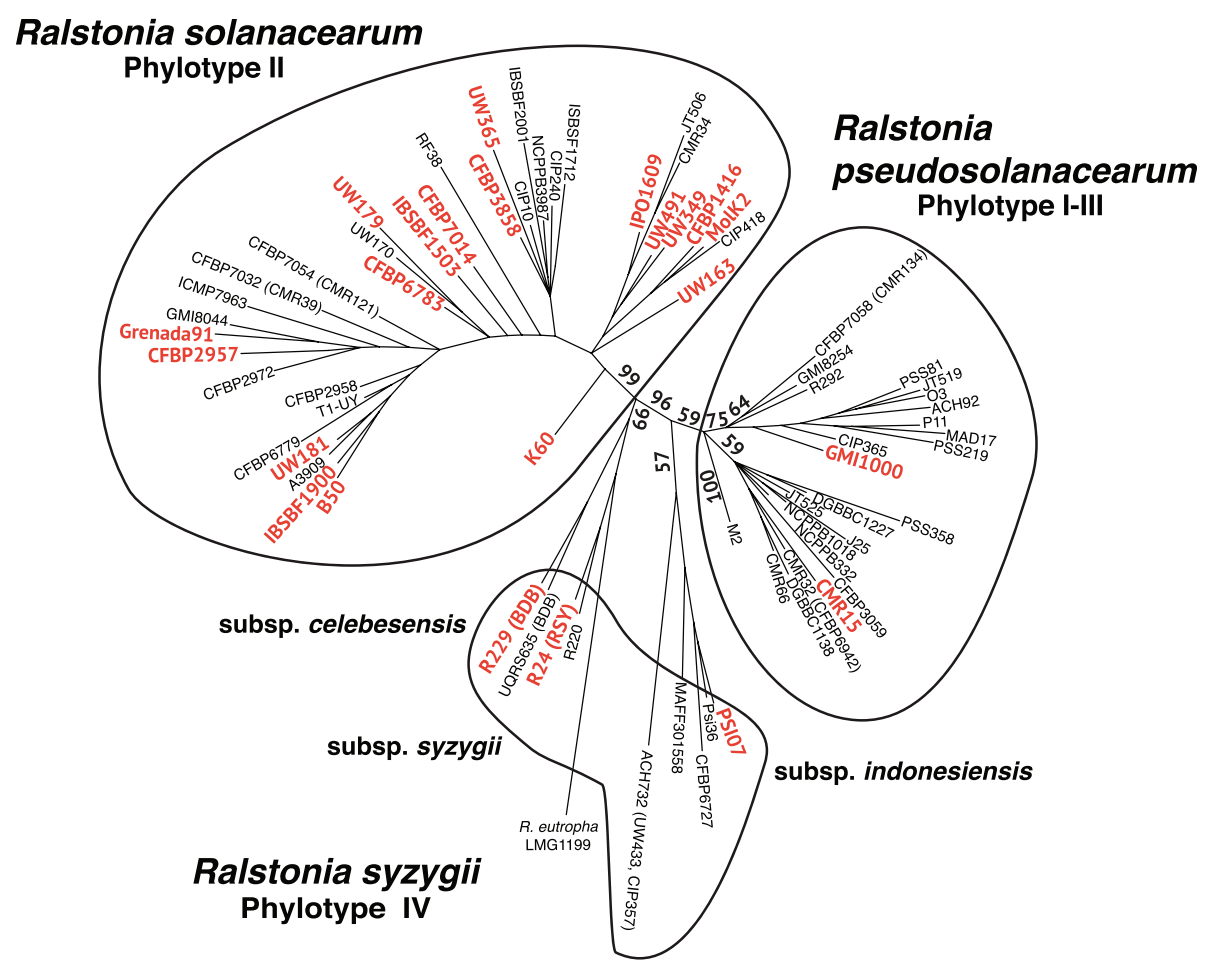

Fig. 3 Tree derived from mass spectrometry analysis. Majority-rule consensus tree based on the presence/absence of a consensus MALDI-TOF peak list obtained using the MrBayes software. The probability values are indicated along the main branches. Red colored strains indicate the sequenced strains. Black lines delineate strain clusters 
variants. Safni et al. recently used DDH for a taxonomic revision of the RSSC, proposing the division of this species complex into three distinct species. We used modern genomic techniques to explicitly reveal the phylogenetic relationships between strains and support a revision of the current taxonomy of this species complex. Moreover, phenotypic data such as measures of inorganic nitrogen metabolism can be directly correlated with genomic content in order to better understand the traits used to delineate species. Using a combination of phenotypic analyses, whole-genome comparisons and proteomic profiling, we provide additional information on the relationships between RSSC strains and offer useful phenotypic tests that distinguish among groups. The first species includes phylotypes I and III. The strains from these two phylotypes undergo denitrification, among other unique phenotypic properties, and are genetically closely related.

The second species corresponds to phylotype II. The strains in this phylotype are somewhat genomically divergent, resulting in the vague delineation of species based on genomic distances. Nonetheless, every strain belonging to phylotype II evaluated to date exhibits similar phenotypic properties and could therefore be considered a single species. The third species comprises: the phylotype IV strains currently classified as $R$. solanacearum; $R$. syzygii, which is transmitted through tube-building Hindola spp. cercopoid insects, with a host range limited to clove trees (Sumatra disease); and $\mathrm{BDB}$, the causative agent of banana wilt diseases in Indonesia. Despite their strikingly different biological lifestyles [44], these strains are genetically related and share core metabolic activities. $R$. solanacearum, $R$. syzygii and BDB have different geographical distributions and pathogenic potential. Moreover, because these groups are easily genetically distinguishable, even when only considering one metabolic pathway-denitrification, the members of this third group could be considered subspecies.

\section{Conclusions}

Extensive biological, phenotypic, and genetic data demonstrate that the RSSC is too diverse to be considered a single species. The modification of the taxonomy of this organism is necessary to recognize three phylogenetically distinct groups with different biological properties and evolutionary relationships. Newly isolated bacterial wilt strains can readily be assigned to the proposed scheme using existing molecular methods [14]. These changes will benefit many different applications, including breeding plant resistance to bacterial wilt, the identification of new pathological variants, management of quarantine containment and the development of diagnostic tests.

\section{Methods}

The sequenced strains used in the present study are listed in Table 1. The strains used for the proteomic analyses are listed in Additional file 2. The phylotype placement of all strains was confirmed using the multiplex PCR method [14].

\section{Genomics}

The complete and assembled genome sequence data used here are publicly available via the MicroScope web interface at www.genoscope.cns.fr/agc/microscope/home/. The Average Nucleotide Identity (ANIb) between genomes was calculated according to Konstantinidis and Tiedje [30], and the genomic distances were obtained after subtracting the ANIb values from 1 . The Maximal Unique Matches index (MUMi) distances between genomes were calculated using the Perl script developed by Deloger et al. [29] using MUMmer genome alignment software [45]. The Genome-to-Genome Distance Calculator (GGDC) was used as previously described [31]. The DDH values were derived from the GGDC distances using formula 2 [31]. A phylogenetic network derived from the distance matrices produced with all three methods was created using SplitsTree4 software [46]. Distances matrices are available in Additional file 1.

\section{Anaerobic inorganic nitrogen metabolism assessments} Nitrate respiration and complete denitrification were assessed using slightly modified VDM medium $[1,47]$. To decrease nitrate-independent anaerobic growth, we used casamino acids instead of yeast extract [22]. Additionally, we omitted nitrate from the base medium. This was done to allow assays to be conducted with and without nitrate under otherwise similar conditions. Where specified, $30 \mathrm{mM} \mathrm{NO}-$ (the concentration found in host plant xylem sap) was added in the form of filter sterilized $\mathrm{KNO}_{3}$ [22]. $1.5 \mathrm{~mL}$ of this modified VDM $\left(+/-\mathrm{NO}_{3}^{-}\right)$was inoculated with a specified bacterial strain to a starting O.D.600 of $\sim 0.001\left(\sim 1 \times 10^{6} \mathrm{CFU} / \mathrm{mL}\right)$. Tubes were incubated without agitation at $28{ }^{\circ} \mathrm{C}$ under anaerobic conditions in a BD GasPak anaerobic system. Seventy-two hours post inoculation, O.D.600 measurements were taken from each culture. Two to four biological replicates were conducted per strain, per treatment $\left(+/-\mathrm{NO}_{3}^{-}\right)$. To determine if nitrate respiration contributed to anaerobic growth, O.D.600 data were compared between + and $-\mathrm{NO}_{3}^{-}$treatments for each strain and depicted as a ratio. A ratio above 1 indicates that the strain grew better anaerobically when provided with $\mathrm{NO}_{3}^{-}$. A ratio of 1 or below indicates that the addition of $\mathrm{NO}_{3}^{-}$did not enhance anaerobic growth, and that the strain did not respire with $\mathrm{NO}_{3}^{-}$under the conditions tested. Additionally, all cultures were visually assessed ( \pm bubbles) over the course of $96 \mathrm{~h}$ for production of dinitrogen gas, the end product of complete denitrification $[1,48]$. 
Table 1 Ralstonia spp. strains used in whole-genome analyses

\begin{tabular}{|c|c|c|c|c|}
\hline Strain & Phy-Seq. & Isolated from & Geographic origin & Acc. \# \\
\hline GMI1000 & I & Tomato & Guyana & GenBank: NC_003295, NC_003296 \\
\hline FQY_4 & । & Soil & China & GenBank: CP004012, CP004013 \\
\hline Y45 & । & Tobacco & China & GenBank: AFWL00000000 \\
\hline IP01609 & IIB-1 & Potato & Netherlands & GenBank: CU914168, CU914166 \\
\hline UW551 & ||B-1 & Geranium & Kenya & GenBank: AAKL00000000 \\
\hline UW349 & IIB-1 & Potato & Brazil & GenBank: JQOI00000000.1 \\
\hline UW365 & |IB-1 & Potato & China & GenBank: JQSI00000000.1 \\
\hline UW491 & IIB-1 & Potato & Colombia & GenBank: JQSH00000000.1 \\
\hline RS2 & IIB-1 & Potato & N/D & EMBL: PRJEB8309 \\
\hline CFBP3858 & IIB-1 & Potato & Netherlands & EMBL: PRJEB8309 \\
\hline MolK2 & IIB-3 & Banana & Philippines & GenBank: CAHW01000040 \\
\hline CFBP1416 & IIB-3 & Plantain & Costa Rica & EMBL: PRJEB7434 \\
\hline CIP417 & IIB-3 & Banana & Philippines & EMBL: PRJEB7427 \\
\hline UW179 & $\| \mathrm{B}-4$ & Banana & Colombia & EMBL: PRJEB7426 \\
\hline UW163 & $\| \mathrm{B}-4$ & Plantain & Peru & EMBL: PRJEB7430 \\
\hline CFBP6783 & |IB-4 & Heliconia & French West Indies & EMBL: PRJEB7432 \\
\hline Po82 & $\| \mathrm{B}-4$ & Potato & Mexico & GenBank: CP002819, CP002820 \\
\hline IBSBF1503 & IIB-4 & Cucumber & Brazil & EMBL: PRJEB7433 \\
\hline CFBP7014 & IIB-59 & Anthurium & Trinidad & EMBL: PRJEB8309 \\
\hline CFBP2957 & $11 \mathrm{~A}-36$ & Tomato & French West Indies & EMBL: FP885897, FP885907 \\
\hline $\mathrm{K} 60^{\top}$ & $\| \mathrm{A}-7$ & Tomato & United States & EMBL: CAGT01000001 \\
\hline Grenada 9-1 & $11 \mathrm{~A}-6$ & Banana & Grenada & EMBL: PRJEB7428 \\
\hline UW181 & $11 \mathrm{~A}-6$ & Plantain & Venezuela & EMBL: PRJEB8309 \\
\hline B50 & $\| A-24$ & Banana & Brazil & EMBL: PRJEB7421 \\
\hline IBSBF1900 & $\| \mathrm{A}-24$ & Banana & Brazil & EMBL: PRJEB8309 \\
\hline CMR15 & III & Tomato & Cameroon & EMBL: FP885895, FP885896 \\
\hline PSI07 & IV & Tomato & Indonesia & EMBL: FP885906, FP885891 \\
\hline BDB R229 & IV & Banana & Indonesia & EMBL: FR854059 to FR854085 \\
\hline R. syzygii R24 & IV & Clove & Indonesia & EMBL: FR854086 to FR854092 \\
\hline
\end{tabular}

\section{Analysis of denitrification genes}

Presence or absence of homologs involved in denitrification were determined in all sequenced strains (Table 1) using the MicroScope web interface and BLAST [49] to look for loci identified in the GMI1000 strain: narG (RSp0974); nosZ (RSp1368); aniA (RSp1503); and norB (RSp1505). Identity values were computed with the $R$ package seqinr [50] after aligning amino-acid sequences with MUSCLE [51].

\section{Bacterial typing using matrix-assisted laser desorption ionization time-of-flight mass spectrometry}

Seventy-three strains belonging to different phylotypes of the RSSC were characterized at the proteomic level using Matrix-Assisted Laser Desorption Ionization Time-Of-Flight Mass Spectrometry (MALDI-TOF MS). Ralstonia eutropha LMG 1199 was included in the analysis as an outgroup (Additional file 2). Bacterial strains were grown on Kelman broth supplemented with agar for $48 \mathrm{~h}$ at $28{ }^{\circ} \mathrm{C}$. For whole-cell protein extraction, $1 \mu \mathrm{L}$ of the bacterial biomass was collected and resuspended in a solution containing $50 \%(\mathrm{v} / \mathrm{v})$ acetonitrile (Acros Organics, Fair Lawn, NJ, USA) and $1 \%(v / v)$ trifluoroacetic acid (Sigma-Aldrich, Saint Louis, MO, USA) in Milli-Q ultrapure water (EMD Millipore Corporation, Billerica, MA, USA). The suspensions were vortexed twice for $10 \mathrm{~s}$ and centrifuged at 20,000 $\mathrm{g}$ for $10 \mathrm{~min}$ at RT. The supernatants were transferred and aliquoted into new tubes and stored at $-20^{\circ} \mathrm{C}$ until further analysis.

One microliter of the bacterial extracts was mixed with $1 \mu \mathrm{L}$ of a saturated solution of $\alpha$-cyano-4-hydroxycinnamic acid (Sigma-Aldrich), which was used as a matrix. The resulting sample/matrix mixture was deposited onto a stainless plate, dried at room temperature, and 
introduced into the MALDI-TOF MS instrument for analysis. The mass spectra profiles were obtained using a bench-top Microflex ${ }^{\mathrm{Tw}}$ MALDI-TOF from Bruker Daltonics, including the Flex Control and Flex Analysis v3.3 software, at the Bacteriology Division of the CHU of St. Pierre, La Réunion. All spectra were obtained in linear positive-ion mode with an $\mathrm{m} / \mathrm{z}$ range of 2000-20,000 Da. Each spectrum was calculated as the sum of 320 accumulated laser shots obtained after a spiral trajectory of the laser. For each sample, two bacterial extracts were obtained and measured in duplicate, and all the spectra were calibrated using a standard preparation of Escherichia coli DH5 $\alpha$, according to Bruker Daltonics.

All bacterial spectra were analyzed using FlexAnalysis software (Bruker Daltonics) to generate peak lists for each strain, and only peaks with a relative intensity greater than $2 \%$ were considered for cluster analysis (Additional file 3). The peak lists were exported to a CSV file, exported to single files using a custom macro and loaded onto the SPECLUST web-service (http://co.bmc.lu.se/speclust/) to obtain a consensus peak list for all strains considered. For the consensus peaks, a peak match score $(\sigma)$ width of \pm 3 Da was considered.

\section{Phylogenetic reconstruction using the MALDI-TOF data}

The consensus peak list was formatted into a sequential Nexus binary file and loaded into MrBayes 3.2.2 software (http://mrbayes.sourceforge.net/). Phylogeny was obtained through Bayesian inference using the restriction data type (two states: absence or presence of a peptide denoted by a 0 or a 1 , respectively), assuming that the frequencies of the two possible states had a Dirichlet $(1.00,1.00)$ prior. Bayesian analysis was performed in two runs using 8 Markov chains and 3,000,000 generations. The potential scale reduction factor implemented in MrBayes 3.2.2 was used as a convergence diagnostic. A majority-rule consensus tree $(50 \%)$ was obtained after discarding $25 \%$ of the initial trees (burn-in $=0.25$ ) generated before the stabilization of the log likelihood values of the data plotted against the number of generations. The trees were subsequently edited using FigTree v1.3.1 (http://tree.bio.ed.ac.uk/software/figtree/).

\section{Availability of supporting data}

Genomes used in this study are available at the following repositories and accession numbers: GMI1000 [GenBank: NC_003295, NC_003296], FQY_4 [GenBank: CP004012, C P004013], Y45 [GenBank: AFWL00000000], IPO1609 [Gen Bank: CU914168, CU914166], UW551 [GenBank: AAKL00 000000], UW349 [GenBank: JQOI00000000.1], UW365 [GenBank: JQSI00000000.1], UW491 [GenBank: JQSH0000 0000.1], RS2 [EMBL: PRJEB8309], CFBP3858 [EMBL: PRJ EB8309], MolK2 [GenBank: CAHW01000040], CFBP1416 [EMBL: PRJEB7434], CIP417 [EMBL: PRJEB7427], UW179
[EMBL: PRJEB7426], UW163 [EMBL: PRJEB7430], CFBP6 783 [EMBL: PRJEB7432], Po82 [GenBank: CP002819 CP00 2820], IBSBF1503 [EMBL: PRJEB7433], CFBP7014 [EMBL: PRJEB8309], CFBP2957 [EMBL: FP885897, FP885907], K60 [EMBL: CAGT01000001], Grenada 9-1 [EMBL: PRJEB74 28], UW181 [EMBL: PRJEB8309], B50 [EMBL: PRJEB742 1], IBSBF1900 [EMBL: PRJEB8309], CMR15 [EMBL: FP88 5895, FP885896], PSI07 [EMBL: FP885906, FP885891], BD B R229 [EMBL: FR854059 to FR854085], R. syzygii R24 [EMBL: FR854086 to FR854092].

\section{Additional files}

Additional file 1: Genomic distance matrices. Pairwise comparisons of 29 sequenced genomes from the $R$. solanacearum species complex using the ANI, MUMi and GGDC methods. The orange, blue and green cells represent strains clustered into species using the criteria specific to each method and corresponding to $70 \%$ DDH. (XLSX $45 \mathrm{~kb}$ )

Additional file 2: Bacterial strains used for proteomic analysis. Phylotype and sequevar classifications for the strains in the $R$. solanacearum species complex were determined as previously described [14]. The sequenced strains are highlighted in green. (XLSX 47 kb)

Additional file 3: MALDI-TOF peak lists. Peak lists extracted from MALDI-TOF spectrum for 73 R. solanacearum strains with FlexAnalysis software (Bruker Daltonics). (ZIP $76 \mathrm{~kb}$ )

\section{Abbreviations}

ANI: average nucleotide identity; GGDC: genome-to-genome distance calculator; BDB: blood disease bacterium; DDH: DNA-DNA hybridization; MALDI-TOF: matrix-assisted laser desorption/ionization-time of flight; MLST: multi locus sequence typing; MUM: maximum unique matches; RSSC: Ralstonia solanacearum species complex.

\section{Competing interests}

The authors declare that they have no competing interests.

\section{Authors' contributions}

The work presented resulted from collaboration between all authors and each author has participated sufficiently in the work to take public responsibility for the content. PP and CA defined the research theme. FA, BD, $\mathrm{BR}$ and BS performed the experiments. All authors drafted the article and participated in revising it critically for intellectual content. Each author gave final approval of the version of the manuscript to be published.

\section{Acknowledgments}

We gratefully acknowledge Boris Vitnazer for providing some of the IIB-1 genomes and Carolee Bull for valuable discussions. This research was supported through INRA, CIRAD, the University of Wisconsin-Madison College of Agricultural and Life Sciences and by the e.b.s federative structure of the Université de la Réunion through the MALDIV grant EBSFIN13-01. The authors would like to thank Aurélie Lajus and Claudine Médigue (Genoscope, France) for technical support and A. Michault from CHU de La Réunion Groupe Hospitalier Sud Réunion for generously providing the MALDI-TOF-MS instrument. Borja Sánchez received funding from the INRA and a European Union AgreenSkills postdoctoral fellowship (grant agreement $n^{\circ}$ 267196).

\section{Author details}

${ }^{1}$ UMR PVBMT Peuplements Végétaux et Bioagresseurs en Milieu Tropical, CIRAD, Saint Pierre, La Réunion, France. ${ }^{2}$ Anses-Plant Health Laboratory (LSV), 7 chemin de I'IRAT, Saint-Pierre, La Réunion, France. ${ }^{3}$ Department of Plant Pathology, University of Wisconsin-Madison, 1630 Linden Drive, Madison, WI 53706, USA. ${ }^{4}$ Department of Plant Health and Environment (SPE), INRA, Paris, France. ${ }^{5}$ Department of Analytical and Food Chemistry, University of Vigo, Ourense, Spain. 
Received: 17 June 2015 Accepted: 26 January 2016 B.

\section{References}

1. Hayward AC, El-Nashaar HM, Nydegger U, De Lindo L. Variation in nitrate metabolism in biovars of Pseudomonas solanacearum. J Appl Bacteriol. 1990:69:269-80

2. Denny T. Plant pathogenic Ralstonia species. In: Gnanamanickam SS, editor. Plant-associated bacteria. Dordrecht, The Netherlands: Springer Netherlands; 2006. p. 573-644.

3. Hayward AC. Biology and epidemiology of bacterial wilt caused by Pseudomonas solanacearum. Annu Rev Phytopathol. 1991;29:65-87.

4. Smith EF. A bacterial disease of tomato, eggplant, and Irish potato (Bacterium solanacearum nov. sp.). USDA Div Veg Physiol Pathol Bull. 1896, 12:191.

5. Yabuuchi E, Kosako Y, Yano I, Hotta H, Nishiuchi Y. Transfer of two Burkholderia and an Alcaligenes species to Ralstonia gen. nov.: proposal of Ralstonia pickettii (Ralston, Palleroni and Doudoroff 1973) comb. nov., Ralstonia solanacearum (Smith 1896) comb. nov. and Ralstonia eutropha (Davis 1969) comb. nov. Microbiol Immunol. 1995;39(11):897-904.

6. Eden-Green S. Banana blood disease. INIBAP Musa disease fact sheet no 3. 1994

7. Gäumann E. Onderzoekingen over de bloedziekte der bananen op Celebes I. Mededelingen van het Instituut voor Plantenziekten. 1921;50:47.

8. Vaneechoutte M, Kampfer P, De Baere T, Falsen E, Verschraegen G. Wautersia gen. nov., a novel genus accommodating the phylogenetic lineage including Ralstonia eutropha and related species, and proposal of Ralstonia [Pseudomonas] syzygii (Roberts et al. 1990) comb. nov. Int J Syst Evol Microbiol. 2004;54(Pt 2):317-27.

9. Li Z, Wu S, Bai X, Liu Y, Lu J, Liu Y, et al. Genome sequence of the tobacco bacterial wilt pathogen Ralstonia solanacearum. J Bacteriol. 2011;193(21):6088-9.

10. Chun J, Rainey FA. Integrating genomics into the taxonomy and systematics of the Bacteria and Archaea. Int J Syst Evol Microbiol. 2014;64(Pt 2):316-24.

11. Richter M, Rossello-Mora R. Shifting the genomic gold standard for the prokaryotic species definition. Proc Natl Acad Sci U S A. 2009;106(45):19126-31.

12. Cellier G, Prior P. Deciphering phenotypic diversity of Ralstonia solanacearum strains pathogenic to potato. Phytopathology. 2010;100(11): 1250-61.

13. Guidot A, Prior P, Schoenfeld J, Carrere S, Genin S, Boucher C. Genomic structure and phylogeny of the plant pathogen Ralstonia solanacearum inferred from gene distribution analysis. J Bacteriol. 2007:189(2):377-87.

14. Fegan M, Prior P. How complex is the "Ralstonia solanacearum species complex". In: Allen C, Prior P, Hayward AC, editors. Bacterial wilt disease and the Ralstonia solanacearum species complex. St Paul, MN: APS Press; 2005. p. 449-61.

15. Prior $\mathrm{P}$, Fegan $\mathrm{M}$. Recent developments in the phylogeny and classification of Ralstonia solanacearum. Acta Horticult. 2005;695:127-36.

16. Castillo JA, Greenberg JT. Evolutionary dynamics of Ralstonia solanacearum. Appl Environ Microbiol. 2007;73(4):1225-38.

17. N'Guessan CA, Brisse S, Le Roux-Nio AC, Poussier S, Kone D, Wicker E. Development of variable number of tandem repeats typing schemes for Ralstonia solanacearum, the agent of bacterial wilt, banana Moko disease and potato brown rot. J Microbiol Methods. 2013;92(3):366-74.

18. Gabriel DW, Allen C, Schell M, Denny TP, Greenberg JT, Duan YP, et al. Identification of open reading frames unique to a select agent: Ralstonia solanacearum race 3 biovar 2. Mol Plant Microbe Interact. 2006;19(1):69-79.

19. Remenant B, de Cambiaire JC, Cellier G, Jacobs JM, Mangenot S, Barbe V, et al. Ralstonia syzygii, the Blood Disease Bacterium and some Asian $R$. solanacearum strains form a single genomic species despite divergent lifestyles. PLoS One. 2011;6(9):e24356.

20. Salanoubat M, Genin S, Artiguenave F, Gouzy J, Mangenot S, Arlat M, et al. Genome sequence of the plant pathogen Ralstonia solanacearum. Nature. 2002:415(6871):497-502.

21. Safni I, Cleenwerck I, De Vos P, Fegan M, Sly L, Kappler U. Polyphasic taxonomic revision of the Ralstonia solanacearum species complex: proposal to emend the descriptions of Ralstonia solanacearum and Ralstonia syzygii and reclassify current $R$. syzygii strains as Ralstonia syzygii subsp. syzygii subsp. nov., R. solanacearum phylotype IV strains as Ralstonia syzygii subsp. indonesiensis subsp. nov., banana blood disease bacterium strains as Ralstonia syzygii subsp. celebesensis subsp. nov. and R. solanacearum phylotype I and III strains as Ralstonia pseudosolanacearum sp. nov. Int J Syst Evol Microbiol. 2014;64(Pt 9):3087-103.
22. Dalsing B, Truchon A, Gonzalez-Orta E, Milling A, Allen C. Ralstonia solanacearum uses inorganic nitrogen metabolism for virulence, ATP production, and detoxification in the oxygen-limited host xylem environment. mBio. 2015:6(2):e02471-14.

23. Achtman M, Wagner M. Microbial diversity and the genetic nature of microbial species. Nat Rev Microbiol. 2008;6(6):431-40.

24. Rossello-Mora R, Amann R. The species concept for prokaryotes. FEMS Microbiol Rev. 2001;25(1):39-67.

25. Janda JM, Abbott SL. $16 \mathrm{~S}$ rRNA gene sequencing for bacterial identification in the diagnostic laboratory: pluses, perils, and pitfalls. J Clin Microbiol. 2007; 45(9):2761-4.

26. Auch AF, von Jan M, Klenk HP, Goker M. Digital DNA-DNA hybridization for microbial species delineation by means of genome-to-genome sequence comparison. Stand Genomic Sci. 2010;2(1):117-34.

27. Rossello-Mora R. Updating prokaryotic taxonomy. J Bacteriol. 2005;187(18):6255-7.

28. Goris J, Konstantinidis KT, Klappenbach JA, Coenye T, Vandamme P, Tiedje JM. DNA-DNA hybridization values and their relationship to whole-genome sequence similarities. Int J Syst Evol Microbiol. 2007;57(Pt 1):81-91.

29. Deloger M, El Karoui M, Petit MA. A genomic distance based on MUM indicates discontinuity between most bacterial species and genera. J Bacteriol. 2009:191(1):91-9.

30. Konstantinidis KT, Tiedje JM. Genomic insights that advance the species definition for prokaryotes. Proc Natl Acad Sci U S A. 2005;102(7):2567-72.

31. Meier-Kolthoff JP, Auch AF, Klenk HP, Goker M. Genome sequence-based species delimitation with confidence intervals and improved distance functions. BMC Bioinformatics. 2013;14:60.

32. Haley BJ, Grim CJ, Hasan NA, Choi SY, Chun J, Brettin TS, et al. Comparative genomic analysis reveals evidence of two novel Vibrio species closely related to V. cholerae. BMC Microbiol. 2010;10:154.

33. Scortichini M, Marcelletti S, Ferrante P, Firrao G. A genomic redefinition of Pseudomonas avellanae species. PLoS One. 2013;8(9), e75794.

34. Kim M, Oh HS, Park SC, Chun J. Towards a taxonomic coherence between average nucleotide identity and $16 \mathrm{~S}$ rRNA gene sequence similarity for species demarcation of prokaryotes. Int J Syst Evol Microbiol. 2014;64(Pt 2):346-51.

35. Konstantinidis KT, Ramette A, Tiedje JM. The bacterial species definition in the genomic era. Philos Trans R Soc Lond B Biol Sci. 2006;361(1475):1929-40.

36. Henz SR, Huson DH, Auch AF, Nieselt-Struwe K, Schuster SC. Whole-genome prokaryotic phylogeny. Bioinformatics. 2005;21(10):2329-35.

37. Alm R, Johansson P, Hjerno K, Emanuelsson C, Ringner M, Hakkinen J. Detection and identification of protein isoforms using cluster analysis of MALDI-MS mass spectra. J Proteome Res. 2006;5(4):785-92.

38. Ronquist F, Huelsenbeck JP. MrBayes 3: Bayesian phylogenetic inference under mixed models. Bioinformatics. 2003;19(12):1572-4.

39. Böhme K, Fernandez-No IC, Barros-Velazquez J, Gallardo J, Cañas B, CaloMata P. Comparative analysis of protein extraction methods for the identification of seafood-borne pathogenic and spoilage bacteria by MALDITOF mass spectrometry. Anal Methods. 2010;2:1941-7.

40. Degand N, Carbonnelle E, Dauphin B, Beretti JL, Le Bourgeois M, SermetGaudelus I, et al. Matrix-assisted laser desorption ionization-time of flight mass spectrometry for identification of nonfermenting gram-negative bacilli isolated from cystic fibrosis patients. J Clin Microbiol. 2008:46(10):3361-7.

41. Murray PR. Matrix-assisted laser desorption ionization time-of-flight mass spectrometry: usefulness for taxonomy and epidemiology. Clin Microbiol Infect. 2010;16(11):1626-30.

42. Ng HJ, Webb HK, Crawford RJ, Malherbe F, Butt H, Knight R, et al. Updating the taxonomic toolbox: classification of Alteromonas spp. using multilocus phylogenetic analysis and MALDI-TOF mass spectrometry. Antonie Van Leeuwenhoek. 2013;103(2):265-75.

43. Sandrin TR, Goldstein JE, Schumaker S. MALDI TOF MS profiling of bacteria at the strain level: a review. Mass Spectrom Rev. 2013;32(3):188-217.

44. Remenant B, Coupat-Goutaland B, Guidot A, Cellier G, Wicker E, Allen C, et al. Genomes of three tomato pathogens within the Ralstonia solanacearum species complex reveal significant evolutionary divergence. BMC Genomics. 2010;11:379.

45. Kurtz S, Phillippy A, Delcher AL, Smoot M, Shumway M, Antonescu C, et al. Versatile and open software for comparing large genomes. Genome Biol. 2004;5(2):R12.

46. Huson DH, Bryant D. Application of phylogenetic networks in evolutionary studies. Mol Biol Evol. 2006;23(2):254-67.

47. Van den Mooter M, Steenackers M, Maertens C, Gossele F, de Vos P, Swings $J$, et al. Differentiation between Xanthomonas campestris pv. graminis (ISPP 
List 1980), pv. phleipratensis (ISPP List 1980) emend., pv. poae Egli and Schmidt 1982 and pv. arrhenatheri Egli and Schmidt 1982, by numerical analysis of phenotypic features and protein gel electrophoregrams. J Phytopathol. 1987:118:135-56.

48. Zumft WG. Cell biology and molecular basis of denitrification. Microbiol Mol Biol Rev. 1997;61(4):533-616.

49. Camacho C, Coulouris G, Avagyan V, Ma N, Papadopoulos J, Bealer K, et al. BLAST+: architecture and applications. BMC Bioinformatics. 2009;10:421.

50. Charif D, Lobry JR. SeqinR 1.0-2: a contributed package to the R project for statistical computing devoted to biological sequences retrieval and analysis. In: Bastolla U, Porto M, Roman E, Vendruscolo M, editors. Structural approaches to sequence evolution: molecules, networks, populations. Springer Verlag. 2007. p. 207-32.

51. Edgar RC. MUSCLE: multiple sequence alignment with high accuracy and high throughput. Nucleic Acids Res. 2004;32(5):1792-7.

Submit your next manuscript to BioMed Central and we will help you at every step:

- We accept pre-submission inquiries

- Our selector tool helps you to find the most relevant journal

- We provide round the clock customer support

- Convenient online submission

- Thorough peer review

- Inclusion in PubMed and all major indexing services

- Maximum visibility for your research

Submit your manuscript at www.biomedcentral.com/submit
Biomed Central 